\title{
MATHEMATICAL PROBLEMS CONNECTED WITH THE BENDING AND BUCKLING OF ELASTIC PLATES
}

\author{
J. J. STOKER
}

1. Introduction. As part of his Gibbs Lecture of last year Theodore von Kármán [5] considered briefly a number of nonlinear problems concerning thin elastic plates. This address will include discussion of linear as well as of nonlinear problems concerning elastic plates, with special stress on a particular aspect of the nonlinear problems. However, it is not my intention to give a more or less complete survey of the literature on the subject; my purpose is rather to present in some detail a few specific leading ideas and points of view and to call attention to a number of unsolved problems.

The mathematical problems connected with thin elastic plates belong with the many problems from mechanics which have interested eminent mathematicians and from which mathematical ideas of fundamental importance have originated. These problems are of at least equal interest in engineering. In fact, a considerable part of the recent interest in the subject of thin plates can be attributed to the need for numerical solutions of problems which arise in the design of the thin-walled structures used in aircraft. The latter problems are in the main nonlinear boundary value problems of an involved type about which almost nothing of a general nature is known, and, aside from the technical difficulties encountered in attempting to obtain numerical solutions, such problems present questions of a purely mathematical nature which require elucidation. The latter part of this address is largely concerned with the mathematical problems which arise in this connection.

The first attempts to formulate mathematically the problem of flexure of thin elastic plates were probably inspired by the experimental researches of Chladni in 1787 on the modes of vibration of thin elastic plates. During the next twenty years a number of unsuccessful attacks were made upon the problem by James Bernoulli the Younger and others. In 1809 the French Academy offered a prize for a theory of the vibrating plate, which, after some controversy, was awarded to Sophie Germain in 1815. Lagrange, who was a member of the prize committee, corrected the theory of Sophie Germain and derived the partial differential equation as we know it now. In

An address delivered before the New York meeting of the Society on February 21. 1941, by invitation of the Program Committee; received by the editors November 6, 1941. 
1822 Cauchy, prompted by his membership on a committee to consider a paper of Navier on plates, derived for the first time the general linear theory of elasticity substantially in its present form. It is noteworthy that a relatively complicated special problem in elasticity had been formulated, in the main correctly, before the general linear theory of elasticity was developed. One is tempted to enlarge upon the fact that mathematicians of those days felt no distinction between pure and applied mathematics and often found inspiration in quite special problems taken from mechanics.

2. The linear bending problem. The next outstanding historical event in the theory of plates occurred in 1850 with the publication of a famous paper by Kirchoff. In order to explain the significance of the work of Kirchoff it is necessary to formulate briefly the problem
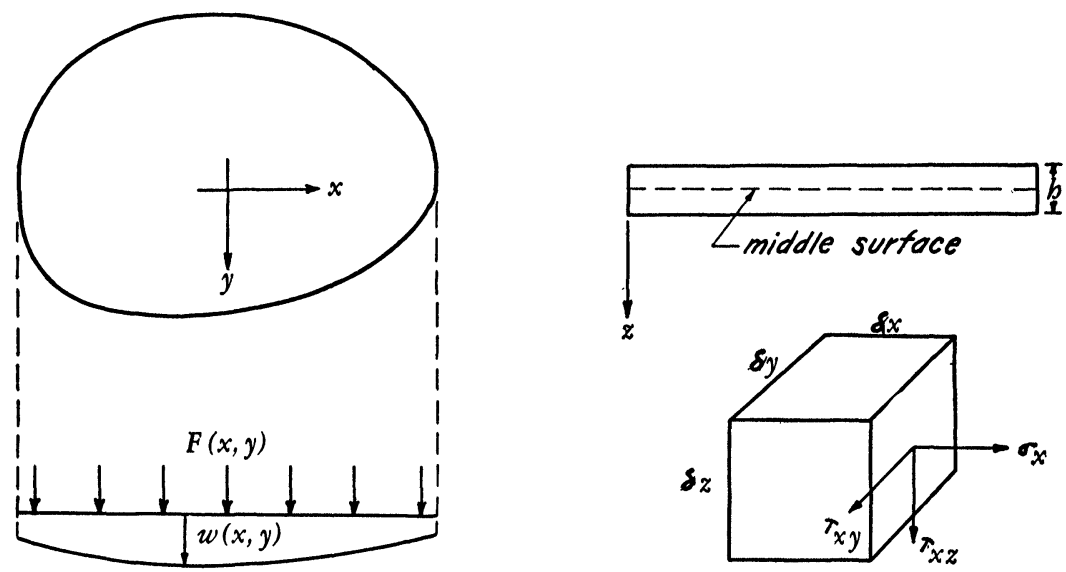

FIG. 1

of the bending of a uniform thin plate subjected to forces perpendicular to the plane of the plate. Assuming that the thickness $h$ of the plate is small in comparison with its other dimensions and that the deflection under load is small in comparison with $h$, it is shown that all stresses in the plate can be calculated in terms of the deflection $w(x, y)$ of the "middle surface" of the plate (Figure 1). The function $w$ is shown to satisfy the linear differential equation:

$$
N \Delta \Delta w=F(x, y), \quad \Delta=\frac{\partial^{2}}{\partial x^{2}}+\frac{\partial^{2}}{\partial y^{2}},
$$

in which $F$ is the force per unit area applied at right angles to the plane of the plate and $N$ is a constant depending only upon the thick- 
ness $h$ and the material of the plate. Once a solution $w(x, y)$ of (1) has been obtained the most important quantities from the practical point of view, that is, the corresponding stresses, can be calculated. More specifically, the normal stress $\sigma_{x}$ and the shear stresses $\tau_{x y}$ and $\tau_{x z}$ (on an element perpendicular to the $x$-axis), for example, are determined from $w$ through the formulas

$$
\begin{aligned}
\sigma_{x} & =\alpha_{1}\left(\frac{\partial^{2} w}{\partial x^{2}}+\nu \frac{\partial^{2} w}{\partial y^{2}}\right), \\
\tau_{x y} & =\alpha_{2}\left(\frac{\partial^{2} w}{\partial x \partial y}\right), \\
\tau_{x z} & =\alpha_{3} \frac{\partial}{\partial x}(\Delta w),
\end{aligned}
$$

where $\alpha_{1}, \alpha_{2}, \alpha_{3}$ depend only upon the thickness of the plate and upon the distance $z$ from the middle surface to the point where the stresses are to be found. The essential point here is that the $\alpha_{i}$ are independent of $x, y$, and $w$. The quantity $\nu$ in (2a) is a material constant called Poisson's ratio.

To solve the problem posed by Chladni's experiments would require the solution of the linear eigenvalue problem associated with (1) (that is, that belonging with the biharmonic equation) under the homogeneous boundary conditions resulting from the assumption that no forces are applied at the edges of the plate. This would mean, one would suppose, that all three of the expressions on the right side of (2) should vanish at an edge perpendicular to the $x$-axis. But the differential equation is of order four, and consequently only two independent boundary conditions can be imposed. This puzzling fact had not escaped the notice of the early workers in the field, but it remained for Kirchoff [9] to settle the question. Kirchoff started from the usual assumptions of the theory, deduced the expression for the potential of the deflected plate, and found by the calculus of variations not only the differential equation (1) but also the correct boundary conditions. At a free edge he found that condition (2a) equated to zero continues to hold but that conditions (2b) and (2c) must be replaced by the single relation (for an element of the boundary perpendicular to the $x$-axis)

$$
q_{x}=\frac{\partial^{3} w}{\partial x^{3}}+(2-\nu) \frac{\partial^{3} w}{\partial x \partial y^{2}}=0 .
$$

This was probably the first striking illustration of the power inherent in the calculus of variations to furnish the correct and appropriate 
boundary conditions for a differential equation. Kirchoff had found what are now called the natural boundary conditions. The idea of natural boundary conditions, when raised to the level of a general concept, has proved to be a fruitful one which has been extensively exploited, for example, in the work of R. Courant and his pupils.

Finally, Kelvin and Tait [8] showed in 1876 how the result of Kirchoff could be reconciled physically with the mechanics of the situation. They observed that conditions $(2 \mathrm{a}, \mathrm{b}, \mathrm{c})$ could all be prescribed for a thick plate and that plausible physical arguments could be advanced indicating that conditions (2b) and (2c) coalesce into (2d) when the thickness of the plate is made small. This aspect of the problem has never been given a rigorous mathematical formulation. It is in fact an example of a "boundary layer" problem-a type of problem to be discussed later in another connection.

A vast amount of work has been done in solving the special linear boundary value problems arising from (1) for plates of various shapes and for various prescribed loading functions $F(x, y)$ and conditions at the boundaries. Many of the cases arising in practice have never been solved in closed form or by series, and resort has been taken to various methods of approximation. One of the most famous of these is the method of Ritz [14]. It is interesting to note that Ritz himself applied his method first to a number of plate problems. Among the problems solved by Ritz is included the first and only solution of the Chladni problem, which had remained unsolved for more than a century. The work of Ritz represents one of the early successes of what are now called direct methods of the calculus of variations. However, the method of finite differences has perhaps proved even more serviceable in obtaining approximate numerical solutions of the linear bending problems [cf. 10].

Although the linear bending problem can be considered as solved in its essentials from the point of view of the mathematician, since the relevant existence and uniqueness theorems are available, there remain nevertheless some unsolved special problems of mathematical interest. For example, there is the curious problem of the rectangular plate resting along its edges on rigid supports and subjected to a constant downward distributed pressure $F$. It is easily shown that a downward concentrated force is necessary at the corners of the plate in order to maintain the edges in contact with the supports; if such forces are not provided, experiments show that the corners will lift up, the plate remaining in contact with the supports only in the central portions of the sides. This presents a boundary value problem of unusual type: The boundary conditions are not given a priori in the 
form of equalities, since the position of the points at which the edge of the plate rises from the supports is not known in advance. The condition $\sigma_{x}=0$ holds everywhere on a boundary line $x=$ const., but there are two alternatives for the other boundary condition, that is, either $w=0$ with $q_{x} \leqq 0$ or $w<0$ with $q_{x}=0$. In spite of this, the problem probably has a unique solution.

3. The von Kármán equations. The linear theory of bending described above was sufficient for most practical purposes until rather recently. With increased use of very thin metal plates, particularly in the structures used in aircraft, it has become of practical importance to obtain solutions based on a theory which would permit
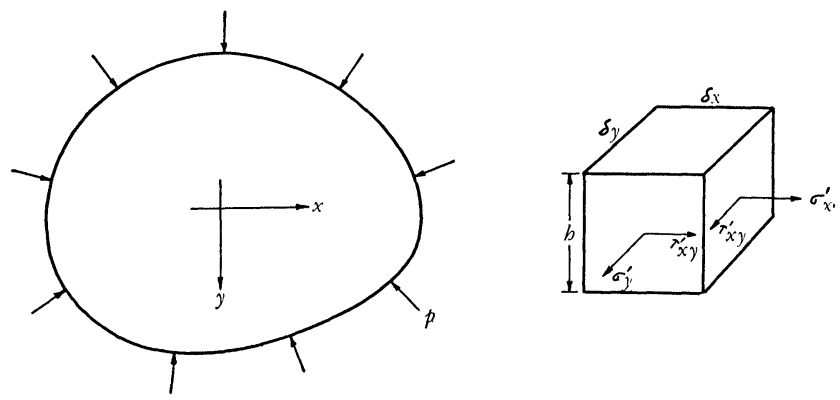

FIG. 2

deflections much greater than the thickness of the plate. The experiments indicate that the linear theory fails to mirror the physical facts if deflections as little as one-fourth of the thickness of the plate occur. We owe to von Kármán [6] an extension of the theory in which the squares of the slopes of the deflected middle surface are not neglected, as is the case with the linear theory. At the same time, the hypothesis of the linear theory that the stresses in the middle surface are zero is given up. The resulting equations of von Kármán are the following pair of fourth order nonlinear differential equations for two functions, the deflection $w(x, y)$ and a function $\phi(x, y)$ from which the stresses in the middle surface, of ten called the "membrane" stresses, are derived:

$$
\begin{aligned}
N \Delta \Delta w & =F(x, y)+h\left\{\frac{\partial^{2} \phi}{\partial y^{2}} \frac{\partial^{2} w}{\partial x^{2}}-2 \frac{\partial^{2} \phi}{\partial x \partial y} \frac{\partial^{2} w}{\partial x \partial y}+\frac{\partial^{2} \phi}{\partial x^{2}} \frac{\partial^{2} w}{\partial y^{2}}\right\}, \\
\Delta \Delta \phi & =E\left\{\left(\frac{\partial^{2} w}{\partial x \partial y}\right)^{2}-\frac{\partial^{2} w}{\partial x^{2}} \frac{\partial^{2} w}{\partial y^{2}}\right\} .
\end{aligned}
$$

The quantity $h$ is the thickness of the plate and $E$ is a material con- 
stant. The stresses $\sigma_{x}^{\prime}, \tau_{x y}^{\prime}, \sigma_{y}^{\prime}$, in the middle surface of the plate (Figure 2) are obtained from $\phi$ by the formulas

$$
\sigma_{x}^{\prime}=\frac{\partial^{2} \phi}{\partial y^{2}}, \quad \tau_{x y}^{\prime}=-\frac{\partial^{2} \phi}{\partial x \partial y}, \quad \sigma_{y}^{\prime}=\frac{\partial^{2} \phi}{\partial x^{2}} .
$$

The boundary conditions for $w$ are linear; they remain the same as for the linear bending problem. At a free edge, for example, the Kirchoff conditions are to be satisfied. Boundary conditions for $\phi$ are obtained from (5) in accordance with whatever "horizontal" forces $p$ are assumed at the boundary of the plate (Figure 2), the term "horizontal" forces being used to distinguish such forces from the "vertical" forces $F$. It is easily shown that this is equivalent to prescribing the values of $\phi$ and its normal derivative $d \phi / d n$ at the boundary of the plate.

4. Buckling problems. A special class of problems is obtained from (3) and (4) by assuming $F \equiv 0$; this we shall do from now on. In other words, there are assumed to be no lateral forces to cause bending. In addition we take always homogeneous boundary conditions for $w$. The problems that arise under these circumstances we refer to as buckling problems.

For the sake of simplicity we assume also that the horizontal forces at the boundary are normal compressive forces which depend linearly on a factor $\lambda$. This is equivalent to taking for $\phi$ the boundary conditions

$$
\phi=\lambda \Phi, \quad d \phi / d n=\lambda \Psi,
$$

where $\Phi$ and $\Psi$ are given functions defined at the boundary of the plate and $\lambda$ is a factor proportional to the applied pressure. Under these circumstances, it is clear that $w \equiv 0$ is always a solution of (3), since $w$ is assumed to satisfy homogeneous boundary conditions. This is also the unique solution for $w$ when $\lambda$ is small enough (in other words, when the applied compressive forces are small enough). However, there is always a critical value $\lambda_{c}$ of $\lambda$ at which the plane state becomes instable and the plate bends, or buckles, in the engineering terminology. Mathematically this means that a bifurcation of the solutions (cf. Figure 4) takes place for $\lambda=\lambda_{c}$ and solutions appear for which $w$ is not identically zero. In this behavior we are reminded of linear eigenvalue problems, though the theory of such problems is naturally not applicable here. Nevertheless it can be shown that the lowest value $\lambda_{c}$ of $\lambda$ for which a bifurcation takes place (or, for which buckling just begins) can always be found from the linear eigenvalue problem which results when the right-hand side of (4) is set equal to 
zero. This implies that the solution $\phi=\lambda \phi_{0}$ of (4) does not depend upon $w$, and hence

$$
N \Delta \Delta w=\lambda h\left\{\frac{\partial^{2} \phi_{0}}{\partial y^{2}} \frac{\partial^{2} w}{\partial x^{2}}-2 \frac{\partial^{2} \phi_{0}}{\partial x \partial y} \frac{\partial^{2} w}{\partial x \partial y}+\frac{\partial^{2} \phi_{0}}{\partial x^{2}} \frac{\partial^{2} w}{\partial y^{2}}\right\}
$$

together with linear homogeneous boundary conditions for $w$ then presents a linear eigenvalue problem from which the lowest value of $\lambda$ for a bifurcation of the solution can be determined. ${ }^{1}$

As in the case of the linear bending problem, a considerable literature has grown up around the eigenvalue problems posed by (6) and many special cases have been treated. Many difficult special cases of practical importance were solved, for example, by Timoshenko [15]; the method used by him bears strong resemblance to the Ritz method. The solutions of these problems have, in general, the properties one is accustomed to associate with linear eigenvalue problems: they possess a discrete spectrum and the eigenfunctions form a complete system in terms of which "arbitrary" functions can be developed.

The problems of existence and uniqueness of the solutions of the eigenvalue problems associated with (6) have been settled in the main. These questions have, however, been reconsidered in recent years by $A$. Weinstein $[17,18]$ from a new point of view. Weinstein has shown that certain classes of plate problems (vibration problems as well as buckling problems) are equivalent to an appropriately chosen sequence of second order membrane problems. An interesting by-product of Weinstein's work is that his method furnishes for the eigenvalues lower bounds which can be calculated numerically.

If a plate is proportioned in such a way that it collapses when compressed by loads only slightly greater than that at which buckling just begins, the linear theory of buckling is evidently sufficient for practical purposes. But very thin metal plates of the type used in various modern structures do not fail even when the applied pressure is many times greater than that at which buckling begins. As a consequence, it is a problem of practical as well as mathematical interest to pursue the solutions of equations (3) and (4) into the nonlinear range for high values of the ratio $\lambda / \lambda_{c}$. In fact, it is highly desirable to investigate if possible the asymptotic behavior of the solutions as $\lambda / \lambda_{c}$ tends to infinity.

The most important case from the practical point of view would be that of the rectangular plate. It is clear that the numerical solution

1 This approach to equation (6) is not the historical one; equation (6) was derived by G. H. Bryan [1] before the von Kármán equations were known. 
of the boundary value problem posed by equations (3) and (4) in this case under prescribed boundary conditions and for all values of $\lambda$ is one of very great difficulty. Some approximate solutions (for example, see [11]) have been given by the energy and perturbation methods, the latter being, roughly speaking, a solution in the form of a power series development with respect to $\lambda-\lambda_{c}$. It is difficult to estimate the accuracy of such solutions, but it seems certain that they are accurate only for rather low values of the ratio $\lambda / \lambda_{c}$.

5. The buckled plate as a "boundary layer" problem. In view of the above remarks, it would seem highly desirable to investigate rigorously and in detail, even if only in a quite special case, the nature of the solutions of (3) and (4), with $F \equiv 0$, in their dependence on $\lambda$ for an unlimited range of values of the ratio $\lambda / \lambda_{c}$. One would then be in a better position to judge the degree of accuracy to be expected in applying approximation methods, such as the perturbation method, to other cases not easily solved explicitly. K. Friedrichs and the author have found and solved such a special case $[2,3,4]$.

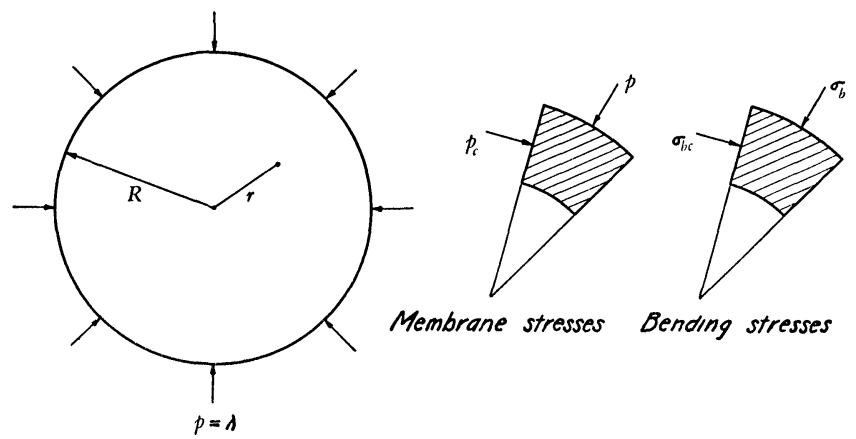

FIG. 3

The special problem in question is that of the circular plate with constant radial pressure applied at the boundary and with radial symmetry assumed (Figure 3 ). In this case all quantities depend only upon the distance $r$ from the center of the plate and the von Kármán equations become ordinary differential equations. ${ }^{2}$ Upon introduction of the quantities $p=(1 / r)(d \phi / d r)$ - the radial membrane stress-and $q=-(R / r)(d w / d r)$ in place of $\phi$ and $w$, respectively, the von Kármán

${ }^{2}$ Solutions of the von Karman equations for the problem of the bending of the circular plate under vertical forces with radial symmetry assumed have been given by Stewart Way [16]. Way found that the von Kármán equations in this case (in spite of their nonlinearity) can be solved conveniently by power series in $r$. 
equations reduce to the following pair of nonlinear differential equations of the second order:

$$
\begin{gathered}
\eta^{2} G q+p q=0, \\
G p=q^{2} / 2,
\end{gathered}
$$

in which $G$ is the linear operator $R^{2} r^{-3} d / d r\left(r^{3} d / d r\right)$. The quantity $\eta^{2}$ occurring in (7) is given by $\eta^{2}=\gamma h / R, h$ and $R$ being the thickness and radius of the plate and $\gamma$ a material constant. At the center $r=0$ the following regularity conditions resulting from the assumed symmetry are imposed:

$$
d q / d r=d p / d r=0
$$

for $r=0$.

At the boundary $r=R$ we assume

$$
\begin{gathered}
R d q / d r+(1+\nu) q=0, \\
p=\lambda>0 .
\end{gathered}
$$

Condition (10) states that the radial bending stress $\sigma_{b}$ is zero at the edge, while the important quantity $\lambda$ in (11) is the prescribed radial pressure at the edge.

Just as in the general case, the only solution of the boundary value problem formulated in equations ( 7$)$ to $(11)$ is $q \equiv 0, p(r) \equiv$ const. $=\lambda$, if $\lambda$ is small enough. The lowest value $\lambda_{c}$ of $\lambda$ for which a different solution appears can be calculated from the linear eigenvalue problem arising from (7) and the homogeneous boundary conditions for $q$, with $p=\lambda$ in (7) as parameter to be determined. The investigation for $\lambda>\lambda_{c}$ requires the solution of the nonlinear boundary value problem as formulated above.

The most striking feature of the numerical solutions in their dependence on $\lambda$ is that $p$ and $q$ (and, in fact, all quantities) appear to tend with increasing $\lambda$ to become constant in an increasingly large portion of the interior of the plate and to change rapidly in a narrow strip, or "boundary layer," near its edge. In addition, $p$ tends to a negative constant in the interior of the plate; physically this means that the compressive stress applied at the edge of the plate (compressive stresses are assumed positive here) results in tension at the center due to buckling and consequent stretch of the plate. To substantiate these statements mathematically it is of course necessary to formulate and solve the limit problem for $\lambda$ tending to infinity.

There are two distinct limit processes involved, the simpler to deal with being that concerning the limit state in the interior of the plate. One obtains the formulation of this problem from the original differ- 
ential equations (7) and (8) by observing that to allow $\lambda$ to tend to infinity for a plate of fixed radius and thickness is mathematically equivalent to allowing the quantity $\eta^{2}=\gamma h / R$ in (7) to tend to zero, $\lambda$ being held fixed. The result is the limit system:

$$
\begin{aligned}
G p & =q^{2} / 2, \\
p q & =0 .
\end{aligned}
$$

The only solution of these equations which satisfies the regularity conditions at $r=0$ is $q \equiv 0, p \equiv$ const. However, the quantity $p$ cannot be set equal to the positive constant prescribed at the boundary, as the value of $p$ in the interior of the plate seems certainly negative in the limit. The limit problem for the interior of the plate is thus not self-contained, since it fails to provide the value to be assigned to $p$ in the limit.

The limit solution for the interior of the plate can be completed only after the limit problem connected with the boundary layer phenomena has been formulated and solved. It should be pointed out that the occurrence of such boundary layer phenomena is not confined to the special problem under consideration here. ${ }^{3}$ Phenomena of the same nature have been noted in various problems concerning thin curved shells (see, for example, [13]). Boundary layer problems occur also in certain nonlinear vibration problems, for example in those concerning what are called relaxation oscillations. All such problems have the same general aspect: Each is a boundary value problem associated with a system of differential equations containing a parameter $\lambda$. A limit solution is desired for a value of $\lambda$ which causes the order of the system of differential equations to degenerate. The consequence is that some of the original boundary conditions are lost, with the result that the convergence as regards $\lambda$ becomes nonuniform at the boundary.

Not all such problems are nonlinear. The problem of KirchoffKelvin-Tait discussed earlier ought to be formulated as a boundary layer problem. The starting point would be the thick plate problem and the limit problem that obtained upon allowing the thickness to approach zero in the differential equations. The differential equations would degenerate and some boundary condition would be lost at the edge. It should be possible to find the nature of the limit state in the boundary layer, at least in some special cases.

No general theory for boundary layer problems exists, but the gen-

${ }^{3}$ The first and most famous boundary layer problem is the one discovered and treated by L. Prandt1 [12] in studying the flow of a viscous fluid around an obstacle. 
eral scheme of attack can be illustrated by means of the case of the buckled circular plate. The solution of the problem in this case, as in others, requires the introduction of a new independent variable in place of $r$ which depends upon the parameter $\lambda$ in such a way that the width of the boundary layer, as measured in the new scale, does not shrink to zero when $\lambda$ tends to infinity. At the same time new dependent variables, replacing $p$ and $q$ and also depending upon $\lambda$, are introduced in such a way that the transformed differential equations resulting from (7) and (8) do not degenerate when $\lambda$ tends to infinity. In short, it is necessary to introduce new variables which have the effect of making the convergence uniform. Limit boundary conditions must also be obtained. The result is in the case of the circular plate a new nonlinear boundary value problem which can be solved to yield limit values for all quantities, including those of most practical importance-the maximum stresses. The maxima of both bending and membrane stresses occur, by the way, in the boundary layer in the limit. Once limit values for all quantities in the boundary layer have been found, we are provided with the particular quantity needed to complete the solution of the interior limit problem-this is, quite naturally, the limit value of $p$ at the inner edge of the boundary layer.

It may be mentioned that the occurrence of the boundary layer phenomena in the case of the circular plate was first observed by solving the original differential equations for a series of increasing values of $\lambda$. Only afterwards were rigorous proofs of the above statements worked out. I should like at this point to call attention to some remarks made by von Kármán in his Gibbs Lecture relative to the need and value, even to those whose interest is mainly a practical one, of existence, uniqueness, and convergence proofs in cases where it is not obvious that the physical phenomena have received a correct mathematical formulation.

Proofs that the limit situation in the buckled circular plate is as described above can be carried out through a confrontation of the boundary value problem with a minimum problem of the following type: The absolute minimum of the functional $V_{\lambda}$ (essentially the potential energy) given by

$$
V_{\lambda}[q]=D[q]-\lambda^{2} H[q]+K[q]
$$

is to be found, where $D, H$, and $K$ are integrals, the first two quadratic in $q$ and $d q / d r$, the third of fourth degree in $d q / d r$. The minimum of $V_{\lambda}$ is sought in the class of all continuous functions $q$ with $L^{2}$-integrable derivatives for which the integrals are finite. It is shown, by direct methods, that the minimum of $V_{\lambda}$ is taken on for a certain such 
function (for each $\lambda$, including $\lambda=\infty$ ) and that each such function possesses a continuous second derivative and furnishes a solution (though by no means all solutions) of the boundary value problem. The relation between the solutions of the minimum and boundary value problems is indicated schematically in Figure 4 which shows the

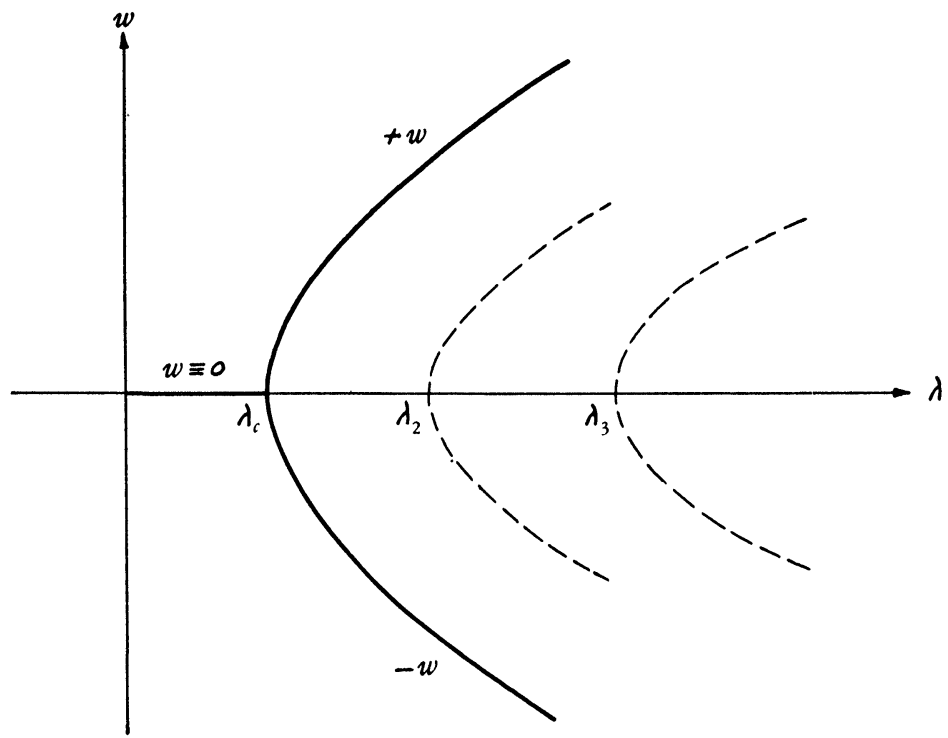

FIG. 4

deflection $w$ of the center of the plate in its dependence on $\lambda$. The heavy curve indicates the type of solution of the minimum problem. (That the solution $-w$ always occurs with $+w$ is readily seen.) However, the boundary value problem has many other solutions, indicated by the dotted curves, in addition to $w \equiv 0$, which is always a solution. The solutions of the boundary value problem which do not vanish identically and which are not solutions of the minimum problem begin at values of $\lambda$ corresponding to the higher eigenvalues of the linearized buckling problem.

6. Some unsolved problems. Quite a number of unsolved questions can be raised at once, even for the special case of the buckled circular plate. For example, it is possible that the solution of the minimum problem eventually becomes instable for a certain value of $\lambda$ and that a new bifurcation arises if the restriction of radial symmetry is given up so that the plate can buckle in waves relative to the original buckled state. Experiments on buckling of rectangular plates indicate 
that such a "second buckling," or buckling of higher order, may well occur. Also, it would be of interest to characterize as minimum problems those solutions of the boundary value problem which begin at the higher eigenvalues of the linearized problem. As is well known, a similar question is solved in the case of linear eigenvalue problems by imposing orthogonality conditions, or by the formulation of a minimum-maximum problem. One could also inquire about the limit state in the case of the circular plate when forces perpendicular to the plane of the plate are applied, with various prescribed boundary conditions. It is known, for example, that the boundary layer effect for the membrane stress does not occur if no compressive stresses arise at the boundary of the plate.

From the practical point of view it would be of high interest to carry the solutions through for the case of the buckled rectangular plate. Even the asymptotic boundary layer solution alone would be of value. The problems with two independent variables seem, however, to be of great difficulty. ${ }^{4}$

One could go on to give a lengthy list of unsolved problems concerning the flexure of plates, some of which have never even been formulated mathematically. For instance, a theory of thin plates analogous to the elastica theory for thin rods ought to be worked out. In such a theory the assumptions of small deflections and slopes of the middle surface would be given up. To complete the analogy with the elastica theory, it should be assumed that the middle surface is unstretched, that is, that it remains a developable surface. In this case the right-hand side of the equation corresponding to (4) would be zero, since the right side of (4) is essentially the Gauss curvature of the middle surface. One would have to pay for this simplification by using the exact expressions for the principal curvatures of the middle surface.

There remain unsolved problems of a purely mathematical character among the classical linear bending problems. The solutions of such problems are in general analytic functions with certain singularities, the character of which is known for a variety, though not all, of the commoner physical situations. The singularity at the corner of a clamped rectangular plate ought to be determined. The numerical solution of the previously mentioned problem of the rectangular

\footnotetext{
${ }^{4}$ Some years ago von Kármán, Sechler, and Donnell [7] gave what can be regarded as a rough approximation to the asymptotic solution for the rectangular plate based on physical assumptions which correspond in a general way to what was found later in the boundary layer theory for the circular plate.
} 
plate resting on rigid supports but with the corners not held down would be greatly facilitated if the singularity at the point of detachment of the plate from its support could be explicitly determined. The best tools for attacking these problems are probably to be found in complex function theory.

A theory with some claim to generality for boundary layer problems would be of high interest. Such a theory should be designed to answer a number of questions. In the first place, one does not know a priori which of the boundary conditions will be lost in a specific problem. This question is coupled with another: In some cases, as in that of the circular plate, it is the boundary layer problem which is selfcontained, while the interior limit problem can be solved completely only after the boundary layer problem has been solved. In the case of the Prandtl boundary layer problem the circumstances are reversed; here the solution of the interior limit problem furnishes a quantity which is needed to complete the solution of the boundary layer problem. It is admittedly not easy to see how a general theory capable of answering such questions should be constructed; probably it is necessary to begin, in the time-honored way, by collecting experience through actual solution of a variety of carefully chosen special problems. Some physical problems likely to be of interest in this connection have already been mentioned-the Kirchoff-Kelvin-Tait problem, for example.

The purpose of the addresses on applied mathematics, of which this is the first, is to stimulate interest in applied mathematics not only for the furtherance of practical ends but also for the advancement of mathematics as a whole. A striking illustration of the beneficial effects on pure mathematics of the study of the applications is certainly provided by the topic of this address. One realizes, then, how great the loss to mathematics in solidity, richness, and variety would be if applied mathematics generally were to be neglected.

\section{REFERENCES}

1. G. H. Bryan, Proceedings of the London Mathematical Society, vol. 22 (1891), p. 54 .

2. K. Friedrichs and J. J. Stoker, The nonlinear boundary value problem of the circu. lar plate, Proceedings of the National Academy of Sciences, vol. 25 (1939), pp. 535540 .

3. - Buckling of the circular plate beyond the critical thrust, Journal of Applied Mechanics, American Society of Mechanical Engineers, (to appear).

4. - The nonlinear boundary value problem of the circular plate, American Journal of Mathematics, vol. 63 (1941), pp. 839-888.

5. T. von Kármán, The engineer grapples with nonlinear problems, this Bulletin, vol. 46 (1940), pp. 615-683. 
6. - Festigkeitsprobleme im Maschinenbau, Enzyklopädie der mathematischen Wissen schaften, vol. IV, 4 (1910), art. 27.

7. T. von Kármán, E. E. Sechler, and L. H. Donnell, Transactions of the American Society of Mechanical Engineers, vol. 54 (1932), pp. 53-57.

8. Kelvin and Tait, Elements of Natural Philosophy, 2d edition, 1879-1883.

9. G. Kirchoff, Journal für die reine und angewandte Mathematik (Crelle), vol. 40 (1850).

10. H. Marcus, Die theorie elastischer Gewebe . . ., Springer, 1923.

11. K. Marguerre and A. Kromm, Verhalten eines Plattenstreifens oberhalb der Beulgrenze, Jahrbuch Deutschen Versuchanstalt für Luftfahrtf, vol. 14 (1937).

12. L. Prandtl, The mechanics of viscous fluids, in Durand: Aerodynamic theory, vol. 13 (1935), $\S \S 13,14$.

13. H. Reissner, Spannungen in Kugelnschalen, Muller-Breslau-Festschrift, 1912.

14. W. Ritz, Gesammelte Werke, pp. 192-316.

15. S. Timoshenko, Sur la stabilité des systèmes élastiques, Annales des Ponts et Chaussées, (1913).

16. S. Way, Bending of circular plates, Transactions of the American Society of Mechanical Engineers, vol. 56 (1934).

17. A. Weinstein, Étude des Équations aux Derivées Partielles de la Théorie des Plaques Élastiques, Mémorial des Sciences Mathématiques, vol. 88 (1937).

18. - Les vibrations et le calcul des variations, Portugaliae Mathematica, vol. 2 (1941), p. 36.

New York UNiversity 